\title{
The Performance of PoolODMRP Protocol
}

\author{
ShaoBin Cai and XiaoZong Yang \\ Harbin Institute of Technology, Department of Computer, Mail-box 320 Harbin Institute of \\ Technology, Harbin, 150001 \\ csb@ftcl.hit.edu.cn
}

\begin{abstract}
The PoolODMRP is proposed as an extension of PatchODMRP[1]. The PoolODMRP reduce the local recovery scope of PatchODMRP to one-hop with help of pool nodes. In the PoolODMRP, the nodes, which are not forwarding nodes, however, can receive data packet, are defined as pool nodes to collect route information from their received data. When a forwarding node detects that there is a link failure between it and one of its upstream nodes, it sends out a Local Query. After receiving Local Query, a neighbor pool node checks whether it connects to the sources defined in the Local Query packet by the route information collected form data packets. If it does, the neighbor answers with a Local Reply. The repairing forwarding node collects the information in its received Local Reply, and informs the oldest answered neighbor pool node to be a forwarding node. In this way, one-hop local recovery is realized in PoolODMRP. The simulation results show that the Pool has a similar data deliver ratio with that of ODMRP [2] and PatchODMRP; its local recovery control overhead is $19.2 \%$ of that of PatchODMRP; its control overhead is only $41.2 \%$ of that of PatchODMRP, and $23.6 \%$ of that of ODMRP; its data overhead is only $64.7 \%$ of that of PatchODMP, and $79.7 \%$ of that of ODMRP.
\end{abstract}

\section{Introduction}

The multicast protocols are the inevitable result of the applications of the Mobile Ad Hoc Network, which can be rapidly deployed without any fixed infrastructure in the hostile environment [3][4][5]. The typical application areas of the ad hoc network, which include battlefields, emergency search and rescue site, require lots of one-tomany and many-to-many communications. Compared with multiple unicasts, the multicast makes full use of the inherent broadcast property of wireless communication, and minimizes the link bandwidth consumption, sender and router processing, and data delivery delay [6].

The already proposed multicast protocols [1][2][7][8][9][10][11] for the ad hoc network can be classified into two categories: tree-based protocols and mesh-based protocols. In the tree-based schemes, a single shortest path between a source and a destination is selected out for the data delivery. MAODV (Multicast Ad-hoc OnDemand Distance Vector), AMRoute (Ad hoc Multicast Routing)[7] and AMRIS (Ad hoc Multicast Routing protocol utilizing Increasing idnumberS)[8] are typical treebased schemes. In a mesh-based scheme, multiple paths are selected for the data delivery. ODMRP (On Demand Multicast Routing Protocol)[2][9][10], PatchODMRP

A. Marshall and N. Agoulmine: MMNS 2003, LNCS 2839, pp. 90-101, 2003.

(C) IFIP International Federation for Information Processing 2003 
[1] and CAMP (Core-Assisted Mesh Protocol)[11] are typical mesh-based schemes. In these protocols, tree-based protocols are generally more efficient than mesh-based protocols, but they are not as robust against topology changes as the mesh-based schemes. The recent studies show that the mesh-based schemes generally outperform the tree-based schemes, and they also show that ODMRP outperforms CAMP both in its protocol efficiency and data delivery ratio [12]. And, the further study shows that PatchODMRP outperforms ODMRP by its two-hop or three-hop local recovery [1]. If we can reduce the scope of local recovery of PatcchODMRP further, then we can reduce its control overhead, and improve its performance greatly.

In the PoolODMRP protocol, the Pool nodes, which are not forwarding nodes, however, can receive data packet, collect route information from their received data packets to provide route information for local recovery. With the help of pool nodes, the PoolODMRP protocol realizes its one-hop local route recovery.

The rest of the paper is organized as follow. First, we give an overview of the ODMRP in section 2. Secondly, we introduce the PatchODMRP in section 3. Thirdly, we describe the PoolODMRP protocol in section 4. And then, we present the simulation results in section 5. Finally, we draw a conclusion in section 6.

\section{Introduction of ODMRP Protocol}

In ODMRP, multicast routes are established and updated by the source on demand. When a multicast source has data packets to send without knowing the routes, it periodically floods a Join Query to the entire network to refresh the membership information and update the routes. When a node receives a new Join Query packet, it firstly stores the source address and the unique identifier of the packet in its Message Cache to detect duplicate; it secondly inserts or updates its Routing Table with the upstream node address in the packet as the next node for the source node; it last decreases the TLL value of the packet by one, and rebroadcasts the packet updated by its address as upstream address when the TTL value is still greater than zero.

A multicast member creates and broadcasts a Join Reply to its neighbors after receiving Join Query packets. After receiving a Join Reply, a node checks whether one of the next address of the received Join Reply matches its own address. If it does, the node marks itself as a forwarding node, and then broadcasts its own Join Reply packet built upon its Routing Table. By the method described above, the Join Reply is propagated by each forwarding node until it reaches the multicast source via the selected path, and the routes from the source to its receivers are constructed or updated by the procedure.

After the forwarding meshes have been founded, the sources broadcast their data to their neighbors. If a node is a forwarding node, then it rebroadcasts its received unduplicated data packet, else it discards its received data packet. By the relay of the forwarding nodes, the data reach all receivers.

In the ODMRP protocol, the group membership information is maintained by soft states. There are lists of the sources of the multicast groups in the members of the multicast groups. The members refresh or update the list by their received Join Query packets. If a node doesn't receive a Join Query packet from a source for a certain amount of time, it deletes the source from its source list. So, when a node wants to join the multicast group as a source, it sends Join Query packets periodically. When a 
node wants to join the group as a member, it replies the Join Query packet that it received with Join Reply. When a node wants to leave the group, it simply stops sending Join Query or Join Reply.

\section{Overview of PatchODMRP Protocol}

PatchODMRP extends ODMRP by two-hop or three-hop local recovery, and prolongs the network-wide flooding period to reduce the control overhead of route maintenance. It sets up its forwarding mesh as ODMRP does. But, in the PatchODMRP, each forwarding node uses forwarding table to store the route information from Join Reply. Therefore, it knows which nodes are its upstream nodes, for which sources it relay data, and the distance between it and the sources.

In the PatchODMRP, each forwarding node detects the statuses of its neighbors by the BEACON of MAC layer [13] to know whether the link between it and one of its upstream nodes is broken. If the link is broken, then the forwarding node floods ADVT packet to do local recovery. The ADVT includes which node sends out the ADVT packet, includes how many times the ADVT packet can be relied, includes which groups the forwarding node belongs to, includes which sources it relays data for, and includes the distances between it and the sources.

When a node receives an ADVT packet, it first records the route information gotten from the ADVT packet. And then, it checks whether it belongs to the same groups that the repairing forwarding node belongs to, checks whether it relay data for the same sources the repairing forwarding node relay for, and checks whether it is nearer to the sources than the repairing forwarding node does. If all these conditions meet, the node answers the ADVT with a PATCH packet. Otherwise, it decreases the Hopcount of ADVT by 1 , and relays the packet when its Hop-count is greater than 0 . The PATCH arrives at the repairing forwarding node by the reverse way of ADVT transmission, and marks the nodes on the way temporary forwarding nodes. After receiving the Patch packets, the repairing forwarding node selects the shortest patch, and informs these temp forwarding nodes of the result. The nodes off the shortest path aren't temp forwarding again.

Fig 1 describes how the PatchODMRP works. The multicast mesh is made up of three fractions (Fig1A): a source (A), a receiver (E), and five forwarding nodes (B, D, F, G, I) marked gray. Node B is the upstream node of node D. When node D can't exchange BEACON with node $\mathrm{B}$, it floods out its ADVT packet. After receiving the ADVT packet, its neighbors $(\mathrm{C}, \mathrm{H}, \mathrm{E})$ update their Route Tables, and relay the ADVT packet (Fig1B). When the forwarding nodes (B, F), which meet the three conditions above, receive an ADVT packet, they answer with a PATCH packet. The nodes $\mathrm{C}$ and $\mathrm{H}$ are marked as temp forwarding nodes by the PATCH packet (Fig1C). After receiving PATCH packets, the repairing forwarding node D selects the shortest path, and informs all these answered forwarding nodes the result, and the node $\mathrm{H}$, that doesn't on the shortest path, isn't a temporary forwarding node again (Fig1D). 

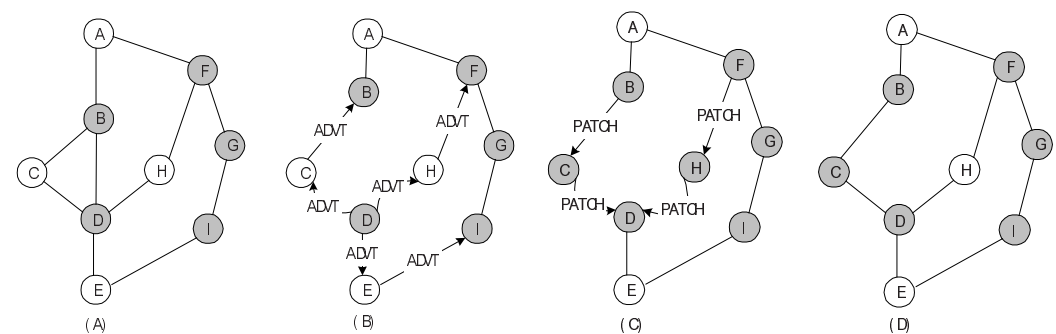

Fig. 1. An example of PatchODMRP

\section{The PoolODMRP Protocol}

In the PatchODMRP, local recovery is performed by two-hop or three-hop scope local flood [1]. If there is a wanted forwarding node two-hop away, the local repair can be completed by marking the node, which is one-hop away from the repairing node, a temp forwarding node. If a node knows from its received packets not only which sources it connects to but also the distance between it and the sources, then we can finish the local repair in one-hop instead of two-hop. And the control overhead of local recovery can be reduced greatly.

In order to realize the one-hop local repair in the PoolODMRP, the pool nodes, which are not forwarding nodes, however, can receive data packets during the data transmission, are defined to collect route information from its received data. Three kinds of new data structure are defined to record the route information that is used for local recovery: Upstream Table (Fig2A), Pool Table (Fig2B) and Candidate Table (Fig2C). And, three new kinds control packets are defined to perform local recovery: Local Query packet (Fig2D), Local Reply packet (Fig2E), and Local Notify packet (Fig2F).

Firstly, we introduce the Upstream Table, which exists in the forwarding node, and the Pool Table, which exists in the Pool node. Both of them are updated by data packets, and are used to record the route information gotten from the data packets. Their Upstream Node field records the address of the node from which the node received data packet; their MG ID field presents which group the data packet belongs to; their Source field presents what nodes originate the data packet; their Count field presents the shortest distance between the source node and the node; their Timer field presents when the data packet is received. The Birth field of the Pool Table presents when the entry is inserted in the table. Each subentry of these tables has a lifetime. When a subentry can't be updated by data packet in time, it expired, and is deleted. When all subentries of an entry are deleted, the entry is deleted too.

Secondly, we introduce the Candidate Table, which temporarily exists in the local repairing forwarding node. When the forwarding node, which is doing local repair, sends out its Local Query, it creates its Candidate Table that exists for a period time. The field of Candidate Table is from the corresponding field of Local Reply packets. When the repairing node receives a Local Reply from one of its neighbor pool nodes, it inserts the pool node address, the list of the MG ID, and the birth time of the local Reply packet in the corresponding fields of its Candidate Table. 


\begin{tabular}{|l|c|c|c|c|}
\hline $\begin{array}{l}\text { Upsitcam } \\
\text { Node }\end{array}$ & $\begin{array}{c}\text { MG } \\
\text { ID }\end{array}$ & Source & Count & Timer \\
\cline { 3 - 5 } & & Source & Count & Timer \\
\cline { 2 - 5 } & MG & Source & Count & Timer \\
\cline { 3 - 5 } & ID & Source & Count & Timer \\
\hline
\end{tabular}

Fig. 2A. Upstream Table

\begin{tabular}{|l|c|c|c|c|c|}
\hline Tpstream & Birth & MGi & Source & Count & Timer \\
\cline { 4 - 6 } & & ID & Source & Count & Timer \\
\cline { 4 - 6 } & & MGi & Source & Count & Timer \\
\cline { 4 - 6 } & & ID & Source & Count & Timer \\
\hline
\end{tabular}

Fig. 2B. Pool Table

\begin{tabular}{|c|c|c|c|}
\hline Pool node & List of $\mathrm{MG}(\mathrm{I})$ & Birth & Lifétime \\
\hline \multicolumn{4}{|c|}{ Fig. 2C. Candidate Table } \\
\hline $\begin{array}{l}\text { Local } \\
\text { Query }\end{array}$ & $\begin{array}{l}\text { List of : } \mathrm{MG} \text { II), } \\
\text { Source, Count; }\end{array}$ & & $\begin{array}{c}\text { LQ } \\
\text { Address }\end{array}$ \\
\hline
\end{tabular}

Fig. 2D. Local Query

\begin{tabular}{|c|c|c|c|c|}
\hline $\begin{array}{c}\text { Locul } \\
\text { Reply }\end{array}$ & $\begin{array}{c}\text { List of } \\
\text { MG ID }\end{array}$ & $\begin{array}{c}\text { LQ } \\
\text { Address }\end{array}$ & Birth & $\begin{array}{c}\text { Pool } \\
\text { Address }\end{array}$ \\
\hline
\end{tabular}

Fig.2E. Local Reply

\begin{tabular}{l|l|l}
\hline Local Notify & List of MG ID & LN Address \\
\hline
\end{tabular}

Fig. 2F. Local Notify

Thirdly, we introduce the Local Query packet, Local Reply packet and Local Notify packet. Their LQ Address is the address of the repairing forwarding node. The List of $\{$ MG ID, Source, Count $\}$ of Local Query packet presents which group the repairing forwarding node belongs to, for which sources the repairing forwarding nodes relay data, and the distance between these sources and the repairing forwarding node. The List of MG ID of both Local Reply and Local Notify present which groups the repairing forwarding node belongs to. The Pool Address of the Local Reply is the address of the pool node that creates the Local Reply. The Birth field of the Local Reply presents the time when the pool node has connections with these required groups. The LN Address of Local Notify is the address of a pool node that will change to a forwarding node.

The PoolODMRP setups up its forwarding mesh by the same way as the ODMRP does too. After setting up its forwarding mesh by the network-wide flooding, the source begins to send out its data packets. When a node receives a data packet, it inserts or updates its Upstream Table or Pool Table by the arithmetic 1 (Appendix 1). If the node is a forwarding node and the data is unduplicated data, then the node relays the packet, else the node discards the packet. So, the nodes collect information used for one-hop local recovery during the data transmission.

In the PoolODMRP, the BEACON signal of the MAC layer is used by a forwarding node to check which nodes are its neighbors. When it finds an upstream node of its Upstream Table isn't reachable, it determines whether it can connect to all the lost sources by other upstream nodes. If it can't, then it forms its Local Query according to the corresponding entry, and sends out the Local Query packet. When one of its neighbors receives a Local Query packet, it firstly deletes the entry, which matches the LQ Address of the Local Query, in its Pool Table; it secondly uses the arithmetic 2 (Appendix 2) to determine whether it creates and sends out a Local Reply to answer the Local Query. If it does, then the node connects to the sources wanted by the repairing forwarding node, and it is not farther to these sources than the repairing forwarding node does. Therefore, when it sets itself a forwarding node, there will be no loop in the new route. 
When the repairing forwarding node receives a Local Reply, it inserts a new entry in the Candidate Table. After a period of time since its receiving its first Local Reply, the node selects the oldest pool node that has the earliest birth time, and sends out a Local Notify to inform the oldest pool node to be a forwarding node. When a pool node receives a Local Notify packet, it sets itself a forwarding node, and acknowledges the Local Notify packet.

Fig 3 describes how the PoolODMRP protocol works. The ad hoc mesh shown in picture 3A consists of 4 fractions: a source $(A)$, a receiver $(E)$, five forwarding nodes (B, D, F, G, I) marked gray, and three Pool nodes (C, H, E) marked gray grid. When the link between node $\mathrm{B}$ and node $\mathrm{D}$ breaks because of the movement of node $\mathrm{B}$, the node D can't exchange BEACON with node B. The node D sends out a Local Query (fig.3B). When its neighbors (C, H, E) receive the Local Query, they check whether they have at least one wanted upstream node. The pool nodes $(\mathrm{C}, \mathrm{H})$, which can receive data from node A, send out their Local Reply to answer the Local Query (fig.3C). After receiving the Local Reply, node D selects the node C out as the oldest pool node and sends out its Local Notify (fig.3D). The node C sets itself a forwarding node as soon as it receives the Local Notify, relays its received data packet, and acknowledges the Local Notify (fig.3E).

\section{The Performance Analyses}

The reason why we use one-hop local recovery is that we want to reduce the control overhead of local recovery. So, we first set up a mathematic model and compare the local control overhead of both PoolODMRP and PatchODMRP. Firstly we assume that a local repairing forwarding node averagely has $\mathrm{L}$ neighbors, $\mathrm{M}$ of which have connections with the wanted forwarding nodes, and has $\mathrm{N}$ wanted forwarding nodes two-hop away. When $C_{\text {pool }}$ is used to represent the local recovery overhead of

PoolODMRP, the cost of $C_{\text {pool }}$ is defined in the equation (1). When $C_{\text {patch }}$ is used to represent the two-hop local recovery overhead of PatchODMRP, the cost of is $C_{\text {patch }}$ defined in (2). And, the huge difference between $C_{\text {patch }}$ and $C_{\text {pool }}$ can be seen in the (3).

$C_{\text {pool }}=1$ (one-hop flooding) $+\mathrm{M}$ (the number of pool nodes which answer the

Join Query)+1 (Local Notify)+1 (ACK for Local Notify),

$C_{\text {patch }}=1$ (one-hop flooding) $+\mathrm{L}$ (the number of nodes which relay the

ADVT) $+\mathrm{N}$ (the number of forwarding which answer the ADVT)+M (the number of pool node which relay the PATCH) $+(1+\mathrm{M})$ (control packet used for confirming the shortest path).

$$
C_{\text {patch }}-C_{\text {pool }}=\mathrm{L}+\mathrm{N}-2
$$



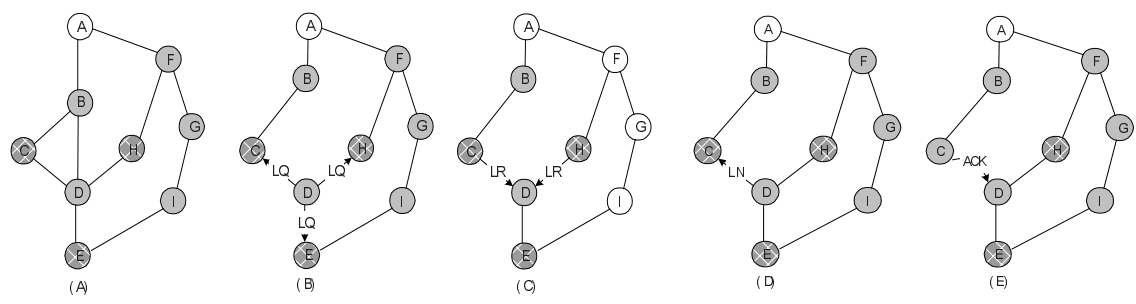

Fig. 3. An example of PoolODMRP

Except the smaller scope of local recovery, PoolODMRP is different from PatchODMRP in other two aspects: the lifetime of the forwarding nodes and the lifetime of the temp forwarding nodes. According to the current PatchODMRP speciation [1], the lifetime of forwarding nodes is set as 3 times as large as the length of the Join Query interval, and the lifetime of temp forwarding nodes is set as 1/3 times as large as the length of the Join Query interval. In the PoolODMRP, there aren't temp forwarding nodes, and the nodes founded in the local recovery are set normal forwarding nodes. The lifetime of forwarding nodes is set as $4 / 3$ times as large as the length of the Join Query interval. The reasons why we reduce the lifetime of the forwarding node not only to reduce the data overhead, but also to reduce unnecessary local recoveries caused by the movement of the too old forwarding nodes. The shorter lifetime of the forwarding node results in not only the lower data overhead and the fewer local recoveries but also the lower data delivery ratio. In order to overcome the shortcomings of the shorter forwarding node lifetime, we use normal forwarding node instead of the temporary forwarding node in the local recovery, and select the most stable route, which is established by the oldest pool node, instead of shortest path in the local recovery of PatchODMRP.

\subsection{Simulation Model}

GloMoSim [14] is used here to realize the simulation of the PoolODMRP protocol. In the simulation, 50 wireless mobile nodes, which move around over a square $(1000 \mathrm{~m} \times 1000 \mathrm{~m})$, form an ad hoc network. The radio transmission power of the mobile nodes is $15 \mathrm{dBm}$. There are two three-member multicast groups in the ad hoc network, one of which has one source, and the other of which has two sources. During the 500s simulation period, Nodes move according to the "random waypoint" model without pause time, and the multicast sources generate 512-byte data packets with constant bit rate (CBR) of ten packets per second. In order to evaluate the performance, we use three metrics here [15]:

Data Packet Delivery Ratio: The percentage of data packets correctly delivered to multicast receivers.

Number of Data Transmissions per Data Packet Delivered: This metric reveals the path efficiency.

Number of Control Packets per Data Packet Delivered: This metric represents control overhead of each data packet. 


\subsection{Numerical Results}

The most important factor, which inflects the performance of PoolODMRP, is its Flood Period, which is the period between two floods. If the Flood Period is longer, the less flooding route discovery operations decrease control overhead, but, the more local unrecoverable link failures may decrease the data delivery ratio. So, we firstly test the impact of the Flood Period on the data delivery ratio and the local recovery ratio. Fig 4 shows the local recovery ratio of PoolODMRP against the changing nodes' max speed. The local recovery ratio is individually $94.9 \%, 97.5 \%$ and $91.4 \%$ when the value of the Flood Period is $12 \mathrm{~s}$, 18s and 24s. Fig 5 shows the average packet delivery ratio of PoolODMRP against the nodes' max speed. The average data delivery ratio is individually $93.5 \%, 95.2 \%$ and $90.4 \%$ when the value of the Flood Period is $12 \mathrm{~s}, 18 \mathrm{~s}$ and $24 \mathrm{~s}$.

According to the theory, the data delivery ratio of the protocol should decrease when the value of Flood Period increases or the nodes' max speed rise. But, the simulation results aren't consistent with the theory because of other factors that affect the data delivery ratio. One of them is the suitable prolonged Flood Period; the other is the suitable movement of the nodes. Because the longer Flood Period decreases the number of network-wide flood, there are less control packets, which contend the scare wireless bandwidth with the data packets. So, when the longer Flood Period doesn't create too many local unrecoverable link failures, it can help data delivery. To illustrate suitable movement benefiting data delivery ratio, let's consider a multicast group composed of nodes A, B and C. When the link between node A and node B breaks as the result of the movement of node $\mathrm{B}$, the node $\mathrm{C}$ can't relay data for node $\mathrm{B}$ because of it can't move close enough to node A and B with the max speed being $5 \mathrm{~m} / \mathrm{s}$. But, the node $\mathrm{C}$ can relay data for node $\mathrm{B}$ because of it can move close enough to node A and B with the max speed being $10 \mathrm{~m} / \mathrm{s}$. Although, the increase of nodes' max speed decreases the data delivery ratio for most time, it may occasionally increase the data delivery ratio.

Form fig4 and fig5, we can see that both the local recovery ratio and data delivery ratio are highest when the value of the Flood Period is $18 \mathrm{~s}$. So, in the flowing experiments, we set the value of the Flood Period of both PatchODMRP and PoolODMRP 18s. And, we set the Flood Period of ODMRP 3s according to the specification of ODMRP [2].

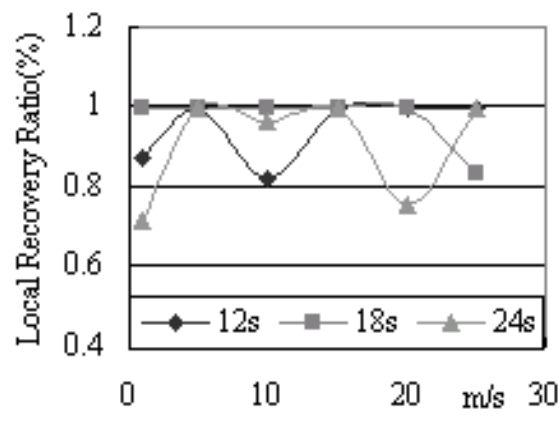

Fig. 4. The relationship between local recovery ratio and the nodes' max speed

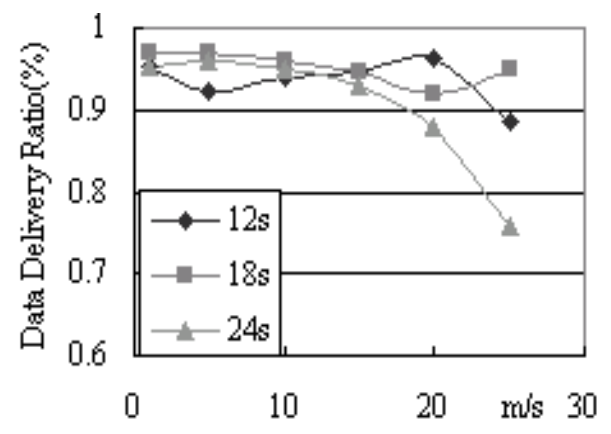

Fig. 5. The relationship between data delivery ratio and the nodes' max speed 
Fig 6 shows the packet delivery ratio of the three protocols against the changing nodes' max speed. The average data delivery ratio of the ODMRP, PatchODMRP and PoolODMRP is respectively $97.1 \%, 96.7 \%$ and $95.2 \%$. The data delivery ratio of PoolODMRP is similar to that of ODMRP and PatchODMRP. According to the definition of both ODMRP and PoolODMRP, the route of ODMRP is more frequently updated because of its lower flood frequency, and there are more forwarding nodes in the forwarding mesh of ODDMRP at most time because of its longer lifetime of its forwarding nodes. Therefore, if there is no local recovery in the PoolODMRP, it is clear that the data delivery ratio of PoolODMRP should be much lower than that of ODMRP. But, the local recovery of PoolODMRP efficiently maintains its forwarding mesh, and PoolODMRP gets a satisfied data delivery ratio. Compared with PatchODMRP, the shorter forwarding nodes' lifetime of PoolODMRP result in its lower data delivery ratio.

Fig 7 shows the average local control overhead of both PatchODMRP and PoolODMRP. The local control overhead is the cost created in the local route recovery procedure. Form the simulation results, we can see that the average local control overhead of both protocols is individually 0.267 and 0.045 , and the local control overhead of PoolODMRP is only $19.2 \%$ of that of PatchODMRP. The smaller local recovery scope of PoolODMRP results in its less local control overhead.

Fig 8 shows the control overhead of the three protocols against the changing node's max speed. From the simulation results, we can see that the average control overhead of ODMRP, PatchODMRP, and PoolODMRP is respectively 0.928, 0.53 and 0.218 . The control overhead of PoolODMRP is only is $23.4 \%$ of that of ODMRP, and $41.2 \%$ of that of PatchODMRP. The control overhead of PatchODMRP and PoolODMRP are both made up of the global control overhead, which are created by the networkwide flooding, and the local control overhead used for the local route recovery. PatchODMRP and PoolODMRP have the similar global control overhead according their definition. So, the lower control overhead of PoolODMRP mainly results from its lower local control overhead. Compared with ODMRP, the longer Flood Period of PatchODMRP and PoolhODMRP results in their lower control overhead.

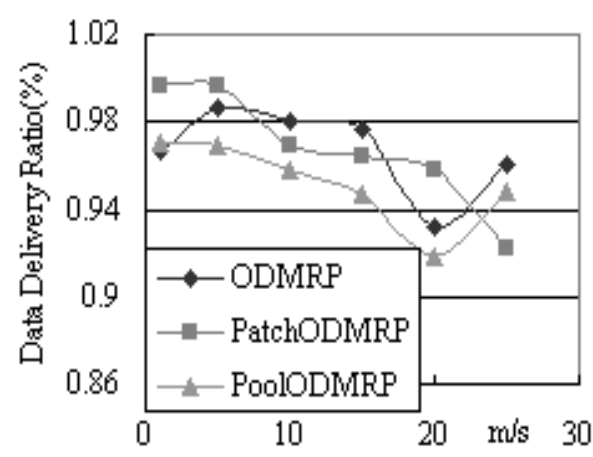

Fig. 6. The relationship between data delivery ratio and the nodes' max speed

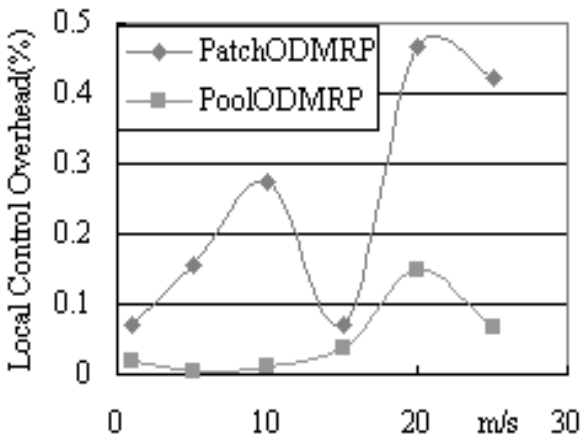

Fig. 7. The relationship between local control overhead and the nodes' max speed 
Fig9 shows the data overhead of the three protocols against the changing node's max speed. The average data overhead of ODMRP, PatchODMRP and PoolODMRP are respectively 2.153, 2.654 and 1.716. The data overhead of PoolODMRP is $79.7 \%$ of that of ODMRP, and is $64.7 \%$ of that of PatchODMRP. The ODMRP and PatchODMRP have the same lifetime of forwarding nodes, so they have the similar data overhead. Since there are repaired routes in the forwarding mesh of PatchODMRP, there are more forwarding nodes in the forwarding mesh of PatchODMRP than that in the forwarding mesh of ODMRP. Therefore, the PatchODMRP has higher data overhead. Compared with ODMRP and PatchODMRP, the forwarding node lifetime of PoolODMRP is shorter. The longer the lifetime of forwarding node, the more forwarding nodes take part in data delivery, the higher data overhead. So, the data overhead of PoolODMRP is lower than that of ODMRP and PatchODMRP.

\section{Conclusion}

In this paper, the PoolODMRP protocol is proposed as a new on-command ad hoc multicast protocol. In the PoolODMRP, the Pool node, which is not a forwarding node, however, can receive data from forwarding nodes, is defined to collect route information from its received data packet. Compared with PatchODMRP, it adopts pool nodes to reduce its local recovery scope, it adopts shorter forwarding node lifetime system to reduce the data overhead, and it adopts normal forwarding node lifetime system in the local recovery to improve its data delivery ratio. The simulation results show that the data delivery ratio of PoolODMRP is similar to that of both ODMRP and PatchODMRP. But, its local recovery control overhead is $19.2 \%$ of that of PatchODMRP; its control overhead is only $41.2 \%$ of that of PatchODMRP, and is $23.4 \%$ of that of PDMRP; its data overhead is only $64.7 \%$ of that of PatchODMP, and is $79.7 \%$ of that of ODMRP. Therefore, PoolODMRP outperforms both ODMRP and PatchODMRP both in control overhead and data overhead.

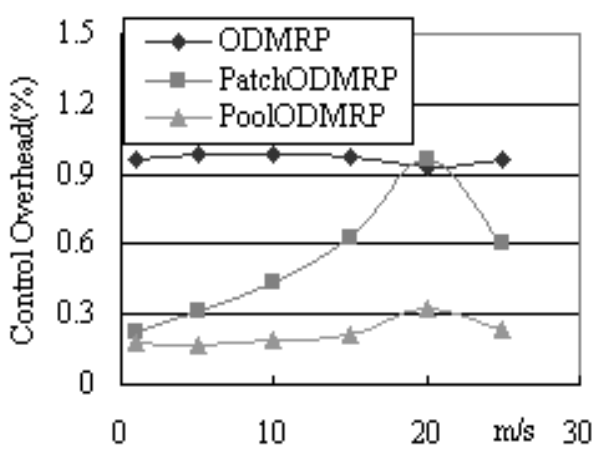

Fig. 8. The relationship between data delivery ratio and the nodes' max speed

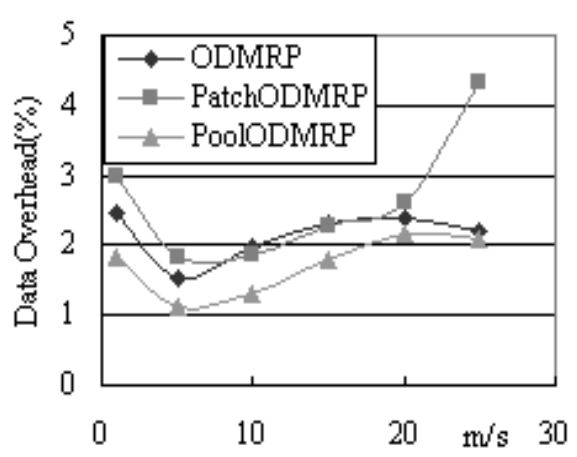

Fig. 9. The relationship between local control overhead and the nodes' max speed 


\section{References}

1. Meejeong Lee; Ye Kyung Kim. "PatchODMRP: an ad-hoc multicast routing protocol" Information Networking, 2001. Proceedings. 15th International Conference on, 2001

2. Sung-ju. Lee, Willian Su, Mario Gerla, "On-Demand Multicast Routing Protocol (ODMRP) for Ad Hoc Networks", Internet Draft, draft-ietf-manetODMRP-02.txt, July 2000.

3. Mobile Ad-Hoc Network (MANET) Working Group [Online]. Available http://www.ietf.org/html.charters/manet-charter.html.

4. D. Bertsekas, R. Gallager, Data Network, second edition, Prentice-Hall, Englewood Cliffs, NJ, 1992, pp. 404-410.

5. C. Perkins, P. Bhagwat, Highly dynamic destination sequenced distance-vector routing (DSDV) for mobile computers, ACM SIGCOMM, October 1994.

6. Sanjoy Paul. "Multicasting on the Internet and its Applications", Kluwer Academic publishers, 1998.

7. E. Bommaiah, M. Liu, A. Mcauley, and R. Talpade, "AMRoute: Ad-hoc Multicast Routing Protocol”, Internet-draft, draft-talpade-manet-amroute-00.txt, Aug, 1998.

8 C.W. Wu, Y.C.Tay, and C.-K.Toh, "Ad hoc Multicast Routing protocol utilizing Increasing id-numberS (AMRIS) Functional Specification”, Internet-draft, draft-ietfmanet-amris-00.txt, Nov 1998.

9. Sung-ju Lee, Willian Su, Mario Gerla, "On-Demand Multicast Routing Protocol”, In proceeding of IEEE WCNC’99. New Orleans, LA, Sep, 1999.

10. Sung-ju Lee, Willian Su, Mario Gerla, "Ad hoc Wireless Multicast with Mobility Prediction”, In proceeding of IEEE WCNC'99. New Orleans, LA, Sep, 1999.

11. J.J. Garcia-Luna-Aceves, E. L. Mdrguga, " The Core-Assisted Mesh Protocol”, IEEE Journal on selected Areas in Communications, vol.17, no. 8, Aug.1999.

12. Sung-ju Lee, Willian Su, Mario Gerla, and Rejive Bagrodia, "A Performance Comparison Study of Ad Hoc Wireless Multicast Protocols”, In Proceeding of Inforcom' 2000, 2000.

13. IEEE Computer Society, LAN MAN Standards Committee. Wireless LAN Medium Access Protocol (MAC) and Physical Layer (PHY) Specification, IEEE Std 802.11-1997 IEEE, New York, 1997.

14. Wireless Adaptive Mobility Lab. DEPT of Comp. SCI, UCLA “ GloMoSim: A Scalable Simulation Environment for wireless and wired Network System:, http://pcl.cs.ucla.edu/projects/domains/glomosim.html.

15. S. Corson and J. Macker. "Mobile Ad Hoc Networking (MANET); Routing Protocol Performance Issues and Evaluation Considerations", RFC 2501, IETF, Jan. 1999, Available at http://www.ietf.org/rfc/rfc2501.txt

\section{Appendix 1 The Arithmetic 1 for Inserting or Updating Upstream Table and Pool Table}

If there is a entry matches last-address of the data packet

\{If there is a subentry matches MG ID of data packet

\{If there is a subentry matches the source of data packet

\{If the Count of the subentry is larger than the Count of data packet

\{Replace the Count field of the subentry with the Count of the data packet;

Update the received time of the data; $\}$

Else \{inserts a subentry to recorder the Source, Count, and the received 
time of the data packet; $\}$ \}

Else \{inserts a new subentry to record the MG ID, Source, Count, and the received time of the data packet; $\}$ \}

Else \{insert a new entry to record the Upstream Node, Birth (only for Pool Table), MG ID, Source, Count, and the received time of the data packet; $\}$

\section{Appendix 2 The Arithmetic 2 for Finding a Matched Upstream Node in the Pool Table}

For each entry of the table

\{If the Upstream Node of the entry doesn't match the LQ address of Local Query

\{For each $\{$ MG ID, Source, Count $\}$ of the list of $\{$ MG ID, Source, Count $\}$

\{If the MG ID of the $\{$ MG ID, Source, Count $\}$ can find a matched subentry in the entry

\{If the Source of the $\{M G$ ID, Source, Count $\}$ can find a matched secondary subentry in the subentry

\{If the Count of the $\{$ MG ID, Source, Count $\}$ is larger than

that of secondary subentry

$\{$ Mark that the $\{$ MG ID, Source, Count $\}$ is satisfied; $\}\}\}\}$

If all $\{\mathrm{MG}$ ID, Source, Count $\}$ of the list of $\{\mathrm{MG}$ ID, Source, Count $\}$ is marked satisfied

\{Create the Local Reply, whose Birth is filled with the Birth of the entry;

Answer the Local Query with Local Reply\};

Break;

\} 